\title{
Soluble ACE2 and angiotensin II levels are modulated in hypertensive COVID-19 patients treated with different antihypertension drugs
}

Huseyin C. Yalcin, Mohamed A Elrayess, Hadeel T. Al-Jighefee, Mahmoud Khatib A. A. Al-Ruweidi, Shamma Almuraikhy, Hadi M. Yassine Biomedical Research Center, Qatar University, PO Box 2713, Doha, QATAR

\section{BACKGROUND}

- Coronavirus disease 2019 (COVID-19) pandemic remains a major threat engendering the lives of millions around the globe, yet effective treatment is still lacking.

- Cardiovascular disease (CVD) is a primary risk factor for the severe acute respiratory syndrome coronavirus 2 (SARS-CoV-2) infection as 2.5-15\% of COVID-19 patients have coronary heart disease and 15-30 $\%$ are hypertensive (1).

- SARS-CoV-2 utilizes the angiotensin-converting enzyme 2 (ACE2) receptor located on the cell membrane to invade the host cells. ACE2 is an essential enzyme in the renin-angiotensin system (RAS), which catalyzes the conversion of angiotensin II into angiotensin 1-7.

- ACE2 could be found in two forms: tissue-specific (membrane-bound) and soluble (circulating) forms. Viral infection, as well as lung and heart diseases, were shown to increase sera-circulating ACE2, partly due to the shedding of the membrane bound ACE-2 (2). The elevation in soluble ACE2 levels in CVD patients was attributed to non-functioning RAS (3).

- In the current clinical practice, different classes of antihypertension drugs are used to control blood pressure in hypertensive patients. The RAS blockers, including angiotensin-converting-enzyme inhibitors (ACEi) and angiotensin receptor blockers (ARB), are used primarily to decrease angiotensin II levels and restore cardiac injury. These drugs can increase ACE2 expression in addition to their role in blocking ACE1 and angiotensin II (4). Therefore, ACEi and ARB were suggested to predispose CVD patients to infection.

- Conversely, other classes of antihypertension drugs such as betablockers (BB) and calcium channel blockers (CCB) exhibit reduced mortality and a putative protective effect in COVID-19 patients with hypertension (5). However, their impact on circulating ACE2, angiotensin II and disease severity remains unknown.

\section{OBJECTIVE}

This multi-center cross-sectional study aims to compare disease severity, circulating ACE2, and angiotensin II levels among COVID-19 hypertensive patients who are treated with $A C E i, A R B, B B$, and CCB. Further, the main objective is to investigate the effect of these drugs on the disease severity in relation to circulating levels of ACE2 and angiotensin II.

\section{METHODS}

Study design: Study involved 200 hypertensive COVID-19 patients (71\% males, $29 \%$ females) recruited from HMC COVID Hospitals. Protocols were approved by the Institutional Review Boards (IRBs) of HMC (MRC-02-20382) and Qatar University (IRB-QU-2020-006). Inclusion criteria require the patients to be hypertensive and under specific and regular antihypertensive medication for at least 30 days before hospital admittance. Patients were categorized into four groups according to taken antihypertension drugs ( $A C E i, n=57, A R B, n=68, B B, n=15$, and $C C B, n=30$ ). RAS blockers included ramipril, perindopril, lisinopril, captopril, candesartan, irbesartan, and losartan. Patients were further categorized into three groups according to their disease severity (mild, $n=76$, moderate, $n=72$, and severe, $n=52$ ). Demographic, anthropometric, and medical history data were collected, including age, ethnicity, vital signs, body mass index (BMI), comorbidities, complete blood count (CBC), Creactive protein (CRP), D-dimer, troponin, kidney, and liver function.

Measurement of circulating ACE2 and angiotensin II: Blood samples were collected within the first three days of hospital admittance. Circulating levels of ACE2 and angiotensin II were measured in the plasma of all participants using validated quenched-fluorescent substrate enzyme linked-immuno-sorbent assays (ELISA).

Statistical analysis: Analysis of clinical traits was carried out using IBM SPSS version 25 and GraphPad Prism version 9.0.1. Variables with skewed distributions (ACE2 and angiotensin II) were log-transformed to ensure normality. Comparisons were performed with t-test, Wilcoxon-MannWhitney, one-way ANOVA, Chi-squared test, and linear regression as appropriate. Significance was defined as $p \leq 0.05$.

\begin{tabular}{|c|c|c|c|c|c|c|}
\hline \multicolumn{7}{|c|}{ RESULTS } \\
\hline & & & & & & \\
\hline & Total $(\mathrm{n}=200)$ & $\operatorname{ACEi}(\mathrm{n}=57)$ & ARB $(\mathrm{n}=68)$ & $\mathrm{BB}(\mathrm{n}=15)$ & CCB $(\mathrm{n}=30)$ & P value \\
\hline Age (years) & $56.2(11.6)$ & $55.7(10.9)$ & $59.7(12)$ & $55.4(11.3)$ & $51.4(10.1)$ & 0.009 \\
\hline BMI $\left(\mathrm{kg} / \mathrm{m}^{2}\right)$ & $30.3(5.3)$ & $30.4(4.7)$ & $30.2(5.5)$ & $32.6(6.5)$ & $-9.2(4.8)$ & 0.2536 \\
\hline Hospital Duration (days) & $14.1(8.7)$ & $13.3(7)$ & $16.1(10.7)$ & $10.2(5.3)$ & $13.3(7.3)$ & 0.05 \\
\hline ACE2 $(\mathrm{gg} / \mathrm{ml})$ & $423.8(610.7)$ & $269.3(267.1)$ & 449.4 (597.4) & $294.1(257.3)$ & $0.2(947.4)$ & 0.008 \\
\hline Angiotensin II ( $(\mathrm{g} / \mathrm{ml})$ & $2784.8(1471.2)$ & $2490.2(1013.4)$ & $3239.6(1873.6)$ & $2844.3(946.8)$ & $2266.8(1061.2)$ & 0.005 \\
\hline $\operatorname{CRP}(\mathrm{mg} / \mathbf{L})$ & $42.1(58.1)$ & $30.1(52.6)$ & $44.0(50.4)$ & $36.9(31.8)$ & $66.2(79.0)$ & 0.0474 \\
\hline D-dimer (mg/L) & $1.8(7.8)$ & $2.6(8.5)$ & $0.64(0.88)$ & $6.1(21.6)$ & $1.9(5.2)$ & 0.1388 \\
\hline Troponin (ng/ml) & $21.1\left(7 \frac{1}{5} .8\right)$ & $20.1(44.2)$ & $10.9(13.8)$ & $7.9(6.2)$ & $15.3(24.2)$ & 0.5642 \\
\hline
\end{tabular}

Figure 1. Circulating ACE2 (A) and angiotensin II (B) levels in COVID-19 hypertensive patients grouped according to disease severity (mild moderate, and severe). Data are presented as dot plots with the mean (95\% Cl). Differences in logtransformed ACE2 and angiotensin I were assessed by ANOVA, ${ }^{*} p<0.05$, ${ }^{* *} p<0.01$

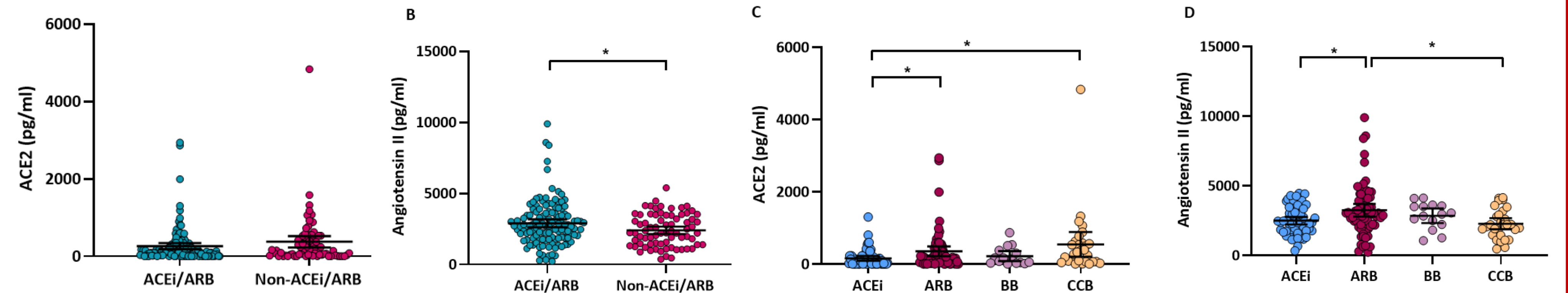

Figure 2. Circulating $A C E 2$ and angiotensin II levels in COVID-19 hypertensive patients grouped according to two (ACEi/ARB, non-ACEi/ARB) (A, B) or four (ACEi, ARB, BB, and CCB) (C, D) medication groups. Differences in log-transformed $A C E 2$ and angiotensin II were assessed by ANOVA, followed by Tukey's post-hoc analysis. ${ }^{*} p<0.05$.
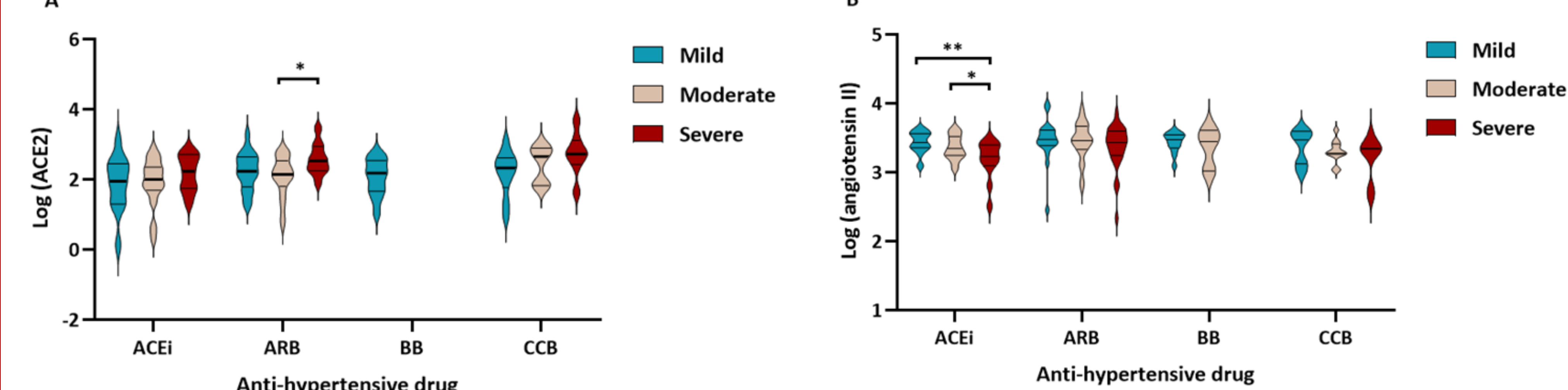

Figure 3. Circulating ACE2 (A) and angiotensin II levels (B) in hypertensive patients with different COVID-19 severity (mild, moderate, or severe) under each medication group (ACEi, ARB, BB, and CCB). Differences in log-transformed ACE2 and angiotensin II were assessed by a linear regression model. $* p<0.05, * * p<0.01$

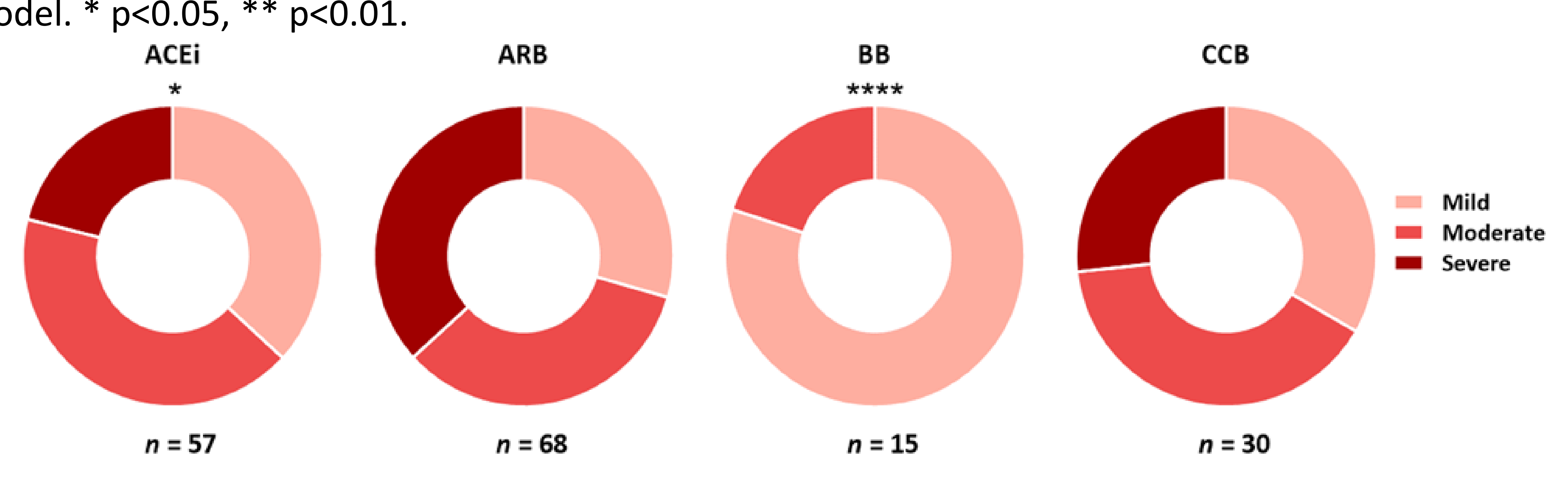

Figure 4. Comparing the percentage of hypertensive patients with different COVID-19 severity under each treatment group. The distribution of patients was compared using the Chi-square test. $\mathrm{p}<0.05, * * * * \mathrm{p}<0.0001$

\section{CONCLUSIONS}

In conclusion, our data highlighted that increased COVID-19 severity is associated with elevated levels of circulating ACE2 and lower levels of angiotensin II and confirmed a potential protective effect of BB treatment against disease severity in hypertensive COVID-19 patients through ACE2 and angiotensin II-independent mechanism.

\section{REFERENCES}

Guo, J., et al., Coronavirus Disease 2019 (COVID-19) and Cardiovascular Disease: A Viewpoint on the Potential Influence of AngiotensinConverting Enzyme Inhibitors/Angiotensin Receptor Blockers on Onset and Severity of Severe Acute Respiratory Syndrome Coronavirus 2

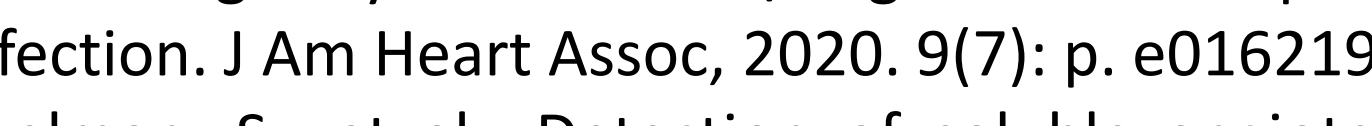

regulatory pathway of the renin-angiotensin-aldosterone system enzyme 2 in heart failure: insights int

hen $L$ et al, The ACE2 expression in human heart indicates new potential mechanism of heart injury among patients infected with SARS-CoV-2. Cardiovascular Research, 2020. 116(6): p. 1097-1100.

Hypertension, 2004. 43(5): p. $970-6$.

Chouchana, L., et al., Association of Antihypertensive Agents with the Risk of In-Hospital Death in Patients with Covid-19. Cardiovasc Drugs Ther, 2021 